\title{
Polycystic echinococcosis in the state of Acre, Brazil: contribution to patient diagnosis, treatment and prognosis
}

\author{
Nilton Ghiotti de Siqueira ${ }^{1 /+}$, Cláudia Maria Villar Maziero de Siqueira², \\ Rosângela Rodrigues-Silva ${ }^{3}$, Manoel do Carmo P Soares ${ }^{4}$, Marinete Marins Póvoa ${ }^{4}$ \\ 'Universidade Federal do Acre, Rio Branco, AC, Brasil ${ }^{2}$ Hospital das Clínicas, Fundação Hospital Estadual do Acre, Rio Branco, AC, Brasil \\ ${ }^{3}$ Laboratório de Helmintos Parasitos de Vertebrados, Instituto Oswaldo Cruz-Fiocruz, Rio de Janeiro, RJ, Brasil \\ ${ }^{4}$ Instituto Evandro Chagas, Secretaria de Vigilância em Saúde, Ministério da Saúde, Belém, PA, Brasil
}

The lack of knowledge regarding polycystic hydatid disease results in delayed or even incorrect diagnosis. The lack of systematic information regarding treatment also makes it difficult to assess the results and prognosis in patients with peritoneal and hepatic lesions caused by Echinococcus vogeli. Here we describe the clinical features of patients, propose a radiological classification protocol and describe a therapeutic option for the treatment of hydatid disease that previously had only been used for cases of cystic echinococcosis (Echinococcus granulosus). A prospective cohort study was initiated in 1999 and by 2009 the study included 60 patients. These patients were classified according to the PNM classification (parasite lesion, neighbouring organ invasion and metastases) and placed in one of three therapeutic modalities: (i) chemotherapy with albendazole at a dose of $10 \mathrm{mg} / \mathrm{kg} / d a y$, (ii) surgical removal of cysts or (iii) percutaneous puncture of the cysts via puncture, aspiration, injection and reaspiration (PAIR). The results were stratified according to therapeutic outcome: "cure", "clinical improvement", "no improvement", "death" or "no information". The PNM classification was useful in indicating the appropriate therapy in cases of polycystic hydatid disease. In conclusion, surgical therapy produced the best clinical results of all the therapies studied based on "cure" and "clinical improvement" outcomes. The use of PAIR for treatment requires additional study.

Key words: polycystic echinococcosis - polycystic hydatid disease -

Echinococcus vogeli - Neotropical echinococcosis - hepatic cysts

Echinococcosis is a zoonosis caused by parasites of the genus Echinococcus. There are four Echinococcus species of medical interest: Echinococcus granulosus, Echinococcus multilocularis, Echinococcus oligarthrus and Echinococcus vogeli (Acha \& Szyfres 1989).

Rausch and Bernstein (1972) described E. vogeli, which is responsible for the polycystic form of echinococcosis and occurs exclusively in the New World (D'Alessandro 1997, D'Alessandro \& Raush 2008). The adult parasite is a tapeworm measuring from $3.9-5.6 \mathrm{~mm}$ that naturally infects the bush dog (Speothos venaticus), which is one of the lowland paca's (Cuniculus paca) natural predators (Raush et al. 1981). The larva transforms into a small cestode in the bush dog's intestine, releasing eggs or egg-containing proglottid eggs into the environment. Lowland pacas are infected while feeding on eggs excreted by the bush dog, which thus completing the life cycle of the parasite (Raush \& D'Alessandro 1999). The hydatid cyst is the morphological expression of the larval form of the disease both in intermediate (lowland paca) and accidental (man) hosts (Acha \& Szyfres 1989).

In the state of Acre (AC), many people inhabit isolated areas that are difficult to reach, where wild ani-

doi: 10.1590/0074-0276108052013001

Financial support: CNPQ/MS, PPSUS/2006, PROJ_569_9269119

+Corresponding author: nilton_ghiotti@uol.com.br

Received 9 January 2013

Accepted 12 April 2013 mal meat, including that of the lowland paca, is often consumed as a protein source. Hunters often recognise hydatid cysts in the liver of the lowland paca and discard the liver. The liver is then eaten by their hunting dogs, which become the definitive hosts of the worm, thus enabling human infection (D'Alessandro et al. 1979, Meneghelli et al. 1990).

Diagnosis is confirmed according to the criteria of D'Alessandro (1997), who proposed an algorithm for the diagnosis of polycystic echinococcosis combining physical examination (presence of abdominal masses), epidemiology (offering of hunting remains to domestic dogs), imaging methods, serology and parasitological diagnosis in a non-systematic review of cases published in South America.

The most common complaint for this infection is upper abdominal pain, but physical signs are nonspecific and secondary to cyst proliferation, which mainly manifests as hardened, palpable abdominal masses of the right hypochondrium with irregular surfaces that are slightly painful to palpation and mobile with respiratory movements. The disease progresses into obstructive jaundice if there is compression of the bile ducts or if biliary fistulas occur. In certain cases, the disease presents as palpable masses in any abdominal quadrant when cysts form in extrahepatic locations; thus, the masses may be mobile to palpation when they are located in the mesentery (Siqueira 2002, Siqueira et al. 2003, 2007, 2010).

The aim of the current study was to evaluate the prognosis and systematise treatment indications in a cohort of patients with hepatic and peritoneal lesions 
caused by E. vogeli. We have also described the patients' clinical conditions and proposed a radiological classification protocol to be used in cases of alveolar echinococcosis and applied this protocol to cases of Neotropical echinococcosis. Additionally, we have characterised the therapeutic option used for the treatment of polycystic echinococcosis: puncture, aspiration, injection and reaspiration (PAIR) (Brunetti et al. 2001).

This project was submitted to and approved by the Research Ethical Committee of the Hospital Foundation of Acre (FUNDHACRE) (Legal Opinion 146/2008).

\section{PATIENTS, MATERIALS AND METHODS}

A prospective cohort was formed beginning in 1999 that included 60 patients by the end of 2009 who were treated at the general surgery outpatient clinic of the FUNDHACRE.

The enrolled patients had positive epidemiological histories for hunting habits and dogs were present in their households. These patients presented with palpable masses in the abdomen and/or ultrasound sonography showing hepatic or peritoneal lesions. The patients were referred for abdominal computed tomography (CT) scan and in cases with typical lesions (multiple cysts with peripheral calcification) (D’Alessandro et al. 1979, Meneghelli et al. 1986), medical treatment was initiated with albendazole at a dose of $10 \mathrm{mg} / \mathrm{kg} /$ day administered orally for three months. After that period, medication was continued for another three months if there were no reports of adverse effects requiring discontinuation of the treatment. The imaging test was repeated and the findings were compared to the previous results to evaluate potential changes in the number or diameter of the cysts and the appearance of calcification, which jointly defined the progression of the disease. At this time, the patient was re-evaluated for possible surgical resection or continuation of chemotherapy treatment with albendazole. The content of cysts obtained from surgery was examined in smears and we identified E. vogeli based the morphology of the rostellar hooks; however, this type of identification was not possible with patients undergoing medical treatment.

From the year 2008 onward, the patients were classified according to the classification system developed by Kern et al. (2006), known as the PNM classification system (parasite lesion, neighbouring organ invasion, metastases) (Supplementary data). This classification system is similar to the tumour-node-metastasis (TNM) staging system of the International Union Against Cancer(UICC 2008) and the American Joint Committee on Cancer(AJCC 2008), in which "T," which refers to the primary tumour location, corresponds to "P," i.e., parasite in the liver parenchyma; "N," which refers to lymph node metastasis, corresponds to "N," i.e., involvement of neighbouring organs and " $\mathrm{M}$ " corresponds to distant metastases in both systems. The patients were classified into stages ranging from "I" to "IV" (Supplementary data), which was similar to the TNM system. However, it was necessary to adapt and create two additional categories or stages to classify cases presenting isolated lesions in the mesentery or retroperitoneum. According to the original classification system, these cases would have been regarded as advanced disease, but this classification would not have correctly represented the clinical presentation or disease prognosis.

Thus, we defined three groups of patients depending on the type of therapeutic approach: (i) patients undergoing medical treatment because the lesions were not resectable, (ii) patients who underwent surgical treatment of isolated mesenteric lesions or resectable hepatic lesions and (iii) patients who underwent percutaneous puncture (PAIR) of voluminous hydatid cysts or cysts with secondary bacterial infection and liver abscess formation.

Qualitative variables regarding treatment outcomes were classified according to the best (cure) and worst (death) outcomes into the following groups: "cure" was defined as the disappearance of lesions after medical or surgical treatment and defined as the best result, "clinical improvement" was used to describe asymptomatic patients without weight loss and with preserved physiological functions, "no improvement" included patients who remained symptomatic, "death" was defined as the worst result and "no information" was used when the variable had not been entered in the research protocol.

\section{RESULTS}

The admission rate of patients to the clinic ranged from one patient/year (2001 and 2004) up to 11 patients/ year (2006 and 2008) (Fig. 1).

The gender distribution showed 1.5 times more men than women ( $336 / 60 ;$ ㅇ $24 / 60)$. The highest frequency of disease progression was observed in stages I (31\%; $18 / 58)$ and IIIb $(41.37 \% ; 24 / 58)$ and the highest incidence was found in the fourth and fifth decades of life $(52.5 \%$; 31/59) (Table I).

Patients were from AC and state of Amazonas (AM). The largest proportion of patients was from Sena Madureira, AC (13/56; 23.2\%), followed by Feijó, AC (7/56; $12.5 \%)$, Tarauacá, AC $(6 / 56 ; 10.7 \%)$ and Brasiléia, AC $(5 / 60 ; 8.9 \%)$.

The habit of hunting was reported by $82 \%$ (41/50) of the patients and dogs were present in the peridomicile in $96 \%(48 / 50)$ of the cases.

Hepatomegaly was detected in $60 \%$ of the cases (33/55), but it was not always observed in conjunction with clinical signs of liver failure, such as jaundice $(17.5 \%, 10 / 57)$,

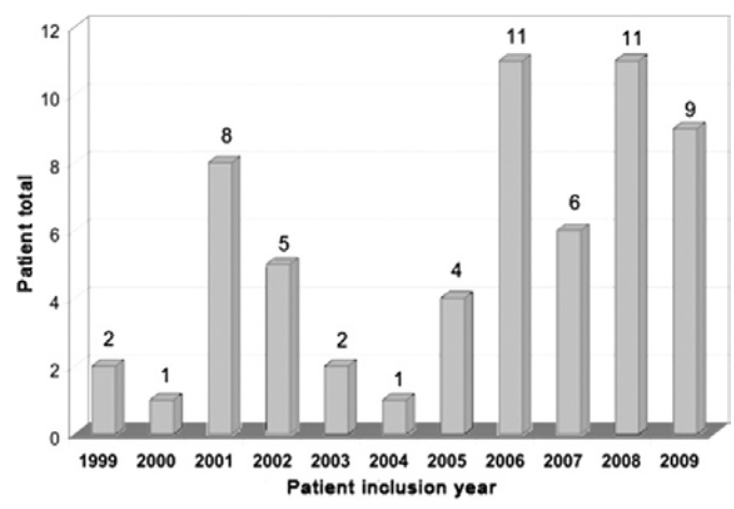

Fig. 1: patients diagnosed with polycystic hydatid disease based on year of enrollment in the study during the period from 1999-2009. 
TABLE I

Distribution of cases of polycystic echinococcosis according to the patients' PNM classification, age and gender who were treated at Hospital Foundation of Acre during the period from 1999-2009

\begin{tabular}{|c|c|c|c|c|c|}
\hline \multirow[b]{2}{*}{ Stage } & & & \multicolumn{2}{|c|}{$\begin{array}{l}\text { Gender } \\
\text { (n) }\end{array}$} & \multirow{2}{*}{$\begin{array}{l}\text { Total } \\
\text { n }(\%)\end{array}$} \\
\hline & & & Female & Male & \\
\hline \multirow[t]{6}{*}{ I } & Age (years) & $21-30$ & 0 & 3 & 3 \\
\hline & & $31-40$ & 1 & 5 & 6 \\
\hline & & $41-50$ & 3 & 2 & 5 \\
\hline & & $51-60$ & 0 & 3 & 3 \\
\hline & & $61-70$ & 0 & 1 & 1 \\
\hline & Total & - & 4 & 14 & $18(31)$ \\
\hline \multirow[t]{3}{*}{ II } & Age (years) & $41-50$ & 0 & 1 & 1 \\
\hline & & $61-70$ & 0 & 1 & 1 \\
\hline & Total & - & 0 & 2 & $2(3.44)$ \\
\hline \multirow[t]{2}{*}{ IIIa } & Age (years) & $41-50$ & 0 & 1 & 1 \\
\hline & Total & - & 0 & 1 & $1(1.72)$ \\
\hline \multirow[t]{9}{*}{ IIIb } & Age (years) & $11-20$ & 3 & 0 & 2 \\
\hline & & $21-30$ & 0 & 3 & 3 \\
\hline & & $31-40$ & 5 & 2 & 7 \\
\hline & & $41-50$ & 0 & 5 & 5 \\
\hline & & $51-60$ & 3 & 0 & 3 \\
\hline & & $61-70$ & 0 & 1 & 1 \\
\hline & & $71-80$ & 0 & 1 & 1 \\
\hline & & $81-90$ & 1 & 0 & 1 \\
\hline & Total & - & 12 & 12 & $24(41.37)$ \\
\hline \multirow[t]{7}{*}{ IV } & Age (years) & $21-30$ & 1 & 0 & 1 \\
\hline & & $31-40$ & 0 & 1 & 1 \\
\hline & & $41-50$ & 1 & 0 & 1 \\
\hline & & $51-60$ & 0 & 1 & 1 \\
\hline & & $61-70$ & 0 & 1 & 1 \\
\hline & & $71-80$ & 1 & 0 & 1 \\
\hline & Total & - & 3 & 3 & $6(10.34)$ \\
\hline \multirow[t]{7}{*}{ Mesenteric } & Age (years) & $0-10$ & 1 & 0 & 1 \\
\hline & & $11-20$ & 1 & 0 & 1 \\
\hline & & $21-30$ & 1 & 0 & 1 \\
\hline & & $31-40$ & 0 & 1 & 1 \\
\hline & & $41-50$ & 0 & 1 & 1 \\
\hline & & $51-60$ & 0 & 1 & 1 \\
\hline & Total & - & 3 & 3 & $6(10.34)$ \\
\hline \multirow[t]{2}{*}{ Retroperitoneum } & Age (years) & $41-50$ & 1 & 0 & 1 \\
\hline & Total & - & 1 & 0 & $1(1.72)$ \\
\hline Total $^{a}$ & - & - & 23 & 35 & $58(100)$ \\
\hline
\end{tabular}

$a$ : 58 because there was an error in the epidemiological form data annotation in two cases; PNM: parasite lesion, neighbouring organ invasion, metastases. ascites $(7.4 \%, 4 / 55)$ or collateral circulation $(5.5 \%, 3 / 55)$. The main signs and symptoms are listed in Table II.

At least one third of the patients showed involvement of both hepatic lobes $(29.5 \% ; 18 / 54)$ on abdominal computed tomography, whereas $10 \%(6 / 60)$ presented mesenteric involvement only and in $1.7 \%(1 / 60)$ the lesion was retroperitoneal. A key diagnostic characteristic was that the lesions showed peripheral calcification in $96.3 \%$ of patients $(52 / 54)$.

The disease was staged according to the PNM classification system in 58 patients (Brunetti et al. 2001). The disease was classified as stage I in $31 \%$ of the patients $(18 / 58)$, stage II in $3.4 \%(2 / 58)$, stage IIIa in $1.7 \%(1 / 58)$, stage IIIb in $41.4 \%(24 / 58)$ and stage IV in $10.3 \%(6 / 58)$, whereas $12 \%(7 / 58)$ fit into the classifications of "mesenteric" $(6 / 58)$ or "retroperitoneal" (1/58) (Table I).

With regard to treatment, $48.3 \%(28 / 58)$ of the patients received only medical treatment with albendazole and $43.1 \%(25 / 58)$ underwent surgery. Of the cases treated with surgical therapy, $48 \%(12 / 25)$ had been previously treated with albendazole. Treatment by percutaneous puncture was performed in $15.5 \%(9 / 58)$ of the cases and all of the patients in this group were treated with 10 $\mathrm{mg} / \mathrm{kg} /$ day of albendazole.

Surgical therapy was combined with percutaneous puncture in four patients. Thus, only five patients $(8.1 \%)$ exclusively underwent PAIR therapy.

In many patients with several years of albendazole use, the lesions remained unchanged relative to the control CT scan (Fig. 2).

The outcomes showed a mortality rate ("death") of $15.5 \%(9 / 58)$ as well as "no improvement" in $1.7 \%(1 / 58)$, "clinical improvement" in 40\% (23/58) and "cure" in $32.8 \%(19 / 58)$ of patients when the three therapeutic groups were analysed jointly.

The total number of patients in the two main groups was similar (albendazole, $\mathrm{n}=28$ and surgery, $\mathrm{n}=25$ ). Regarding the outcome of "death", there was also no difference, with four and three deaths observed per group, respectively. However, in the albendazole group the outcome "cure" was observed in $4.3 \%(1 / 23)$ of cases and "clinical improvement" in 74\% (17/23), whereas in the surgery group, "cure" was observed in 71\% (17/24) and "clinical improvement" in 16.7 (4/24) of the cases (Table III).

\section{TABLE II}

Main signs and symptoms shown by patients with polycystic echinococcosis included in the study, Rio Branco, state of Acre, 1999-2009

\begin{tabular}{lcc}
\hline Signs and symptoms & (n) & $(\%)$ \\
\hline Upper abdominal pain & $39 / 60$ & 65 \\
Palpable liver & $33 / 55$ & 60 \\
Jaundice & $10 / 57$ & 17.5 \\
Extrahepatic mass (es) & $7 / 55$ & 12.7 \\
Oedema & $5 / 54$ & 9.3 \\
Ascites & $4 / 55$ & 7.4 \\
Backache & $4 / 60$ & 6.7 \\
Collateral circulation & $3 / 55$ & 5.5 \\
\hline
\end{tabular}


Comparing the indications for types of therapy among the groups, there was a greater indication for clinical therapy with albendazole in patients at a more advanced stage of the disease and greater indication for surgery in cases staged with less involvement (Table V). Patients classified in stage I were 2.2 times more likely to undergo surgical treatment than clinical treatment and patients classified in stage IIIb received surgical treatment 3.2 times more frequently than clinical treatment.

Regarding the relationship between outcome and classification, "cure" was most frequent in cases of mesenteric and retroperitoneal presentation $(100 \%$; $6 / 6$ and $1 / 1)$ and in patients in stage I $(39 \% ; 7 / 18)$ and the worst outcome, "death", was observed most frequently in patients in stage IIIb $(29.2 \%$; $7 / 24)$ (Table III). The other two deaths occurred at stages I (5.5\%; $1 / 18)$ and IV $(16.7 \% ; 1 / 6)$. There were no deaths in the group with cysts in the mesentery and retroperitoneum.

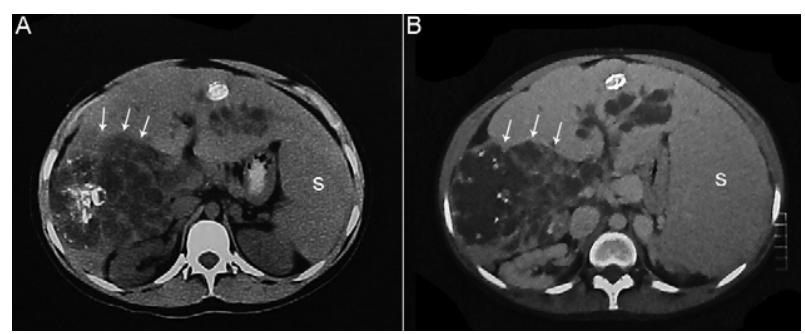

Fig. 2A: image of the abdominal computed tomography, patient, female, 20 years old, resident in the municipality of Sena Madureira, state of Acre, at the onset of chemotherapy with albendazole. Arrows indicate multiple hepatic cysts found during an examination performed in 2001; B: same patient in 2009 (after 8 years of treatment with albendazole). Note the considerable increase of the spleen (S) and the presence of multiple cysts in the right lobe with no changes relative to the previous exam (arrows).
"Clinical improvement" was observed in $39.6 \%$ of the patients (23/58).

At stage I, which demonstrated the best outcomes with respect to presentation of hepatic cysts, the sex distribution was not uniform; there were 3.5 times more males than females, whereas the sex ratio was equal in the other stages.

The follow-up of patients in this cohort ranged from zero-3,755 days, with an average of $884.25 \pm 1,001.81$ days. Three groups were analysed, i.e., patients who underwent treatment with albendazole, surgery and PAIR (Table IV) and the mean, median, minimum, maximum and standard deviation were calculated in days. The value "zero" was assigned to patients who did not return to the outpatient clinic for re-evaluation and these patients were regarded as lost to follow-up. Cases of death during hospitalisation were also assigned a value of "zero." This situation occurred twice in the surgery group. In addition to these two cases, loss of follow-up occurred in 10 patients, including three from the surgery group and seven from the albendazole group. Among the three losses from the surgery group, two patients had isolated lesions, one patient had a mesenteric lesion and the last patient had a retroperitoneal lesion.

\section{DISCUSSION}

Patients were admitted into this cohort over the course of 11 years. Several factors contributed to late diagnosis of polycystic echinococcosis in these patients. Access to referral services through the Brazilian National Health System is unreliable and the difficulty of transit by land during the "Amazonian winter" and the distribution of the hydrographic basin of the $\mathrm{AC}$ hinder travel from the interior to the capital (Rio Branco) by this alternative route. Furthermore, most patients in this study were in good general condition; although they often showed large hepatic masses $(60 \%$ of cases with palpable liver masses), the patients did not exceed the lower

TABLE III

Relationship between disease stage, type of treatment and outcome in participating patients, 1999-2009

\begin{tabular}{|c|c|c|c|c|c|c|c|c|c|c|c|c|c|c|c|c|}
\hline \multirow[b]{2}{*}{ Outcome } & \multicolumn{5}{|c|}{ Albendazole } & \multicolumn{5}{|c|}{ Surgery } & \multicolumn{5}{|c|}{$\operatorname{PAIR}^{a}$} & \multirow[b]{2}{*}{ (n) } \\
\hline & $\mathrm{D}$ & $\mathrm{C}$ & $\mathrm{CI}$ & NI & ND & $\mathrm{D}$ & $\mathrm{C}$ & CI & NI & ND & $\mathrm{D}$ & $\mathrm{C}$ & CI & NI & ND & \\
\hline \multicolumn{17}{|l|}{ Stage } \\
\hline I & - & - & 4 & - & 1 & 1 & 7 & 3 & - & - & - & (1) & 2 & - & - & 18 \\
\hline II & - & - & 1 & - & 1 & - & - & - & - & - & - & - & - & - & - & 2 \\
\hline IIIa & - & - & 1 & - & - & - & - & - & - & - & - & - & - & - & - & 1 \\
\hline $\mathrm{IIIb}$ & 3 & - & 10 & 1 & 2 & 2 & 2 & - & - & 1 & $(1)+2$ & $(1)+1$ & - & - & - & 24 \\
\hline IV & 1 & 1 & 1 & - & 1 & - & 1 & 1 & - & - & - & - & (1) & - & - & 6 \\
\hline Mesenteric & - & - & - & - & - & - & 6 & - & - & - & - & - & - & - & - & 6 \\
\hline Retroperitoneal & - & - & - & - & - & - & 1 & - & - & - & - & - & - & - & - & 1 \\
\hline Sub-totals & 4 & 1 & 17 & 1 & 5 & 3 & 17 & 4 & - & 1 & 2 & 1 & 2 & - & - & - \\
\hline Total & & & 28 & & & & & 25 & & & & 5 & & & & 58 \\
\hline
\end{tabular}

$a$ : although percutaneous punctures were performed in nine patients, we could only consider the treatment through puncture, aspiration, injection and re-aspiration (PAIR) in five. The other four patients (numbers between brackets) underwent more than one procedure and therefore were not considered. C: cure; CI: clinical improvement; D: death; ND: no data; NI: no improvement. 
limit of the liver's functional reserve. Thus, evidence of liver failure, such as jaundice, ascites or collateral circulation, was rarely found during physical examination of the patients, except in cases of advanced disease. This lack of symptoms was perhaps the main reason for the patients' delay in seeking medical care.

Clinically, the highest frequency of infection in the fourth and fifth decades of life indicates the long duration of infection and disease before signs or symptoms are triggered that lead the individual to seek medical help. Only one patient in the study was in the first decade of life and even in this case, the patient had mesenteric presentation without liver involvement.

These findings raise another key issue, which is the lack of knowledge concerning the appearance of such lesions in their early stages on the various imaging tests. Knowledge of the appearance of the lesions during the early stage may lead to earlier diagnosis and improve the clinical and surgical management of this disease.

Most patients in this cohort were from Sena Madureira. Two factors may have contributed to the inclusion of a greater number of patients from this city: (i) knowledge of the disease provided by the seroepidemiological survey conducted by Pastore (2001), during which 851 interviews were held in the urban area and 213 in the rural area in a population of 29,412 inhabitants and (ii) the availability of land transportation during all seasons from that municipality to Rio Branco.

Additionally, the four municipalities with the most cases in AC (Sena Madureira, Feijó, Tarauacá and Cruzeiro do Sul) border AM, from which only two cases have been reported in meeting abstracts (Guimarães et al. 2005).

Similarly, the municipalities of Cruzeiro do Sul, Brasiléia and Epitaciolândia (AC) border Peru and Bolivia, respectively. There is only one published case of polycystic hydatid disease in the Peruvian Amazon Region (Somocurcio et al. 2004) and there are no published cases in Bolivia. Thus, there are likely many undiagnosed or unreported cases in this vast region of Latin America.

Most of these patients have lived on rubber plantations and later migrated to urban centres, which are actually small cities. Even after migration, their habits are highly similar to those that they previously had in the forest, including hunting and consuming game meat $(82 \%)$ and rearing dogs $(96 \%)$, which are the definitive hosts of the disease for transmission to man.
In a secondary review of data from the literature (Siqueira 2002), several differences regarding the prevalence of symptoms and signs between those studies and the present cohort, including abdominal pain $(92.5 \%$ vs. $65 \%$, respectively), jaundice (75\% vs. $17.5 \%$ ) and hepatomegaly ( $70.6 \%$ vs. $60 \%)$, were found, but these signs and symptoms were not always reported in the original studies reviewed by the secondary study, which resulted in a lower number of patients with available information.

Among imaging tests, ultrasonography is the method of choice in epidemiological surveys because of its low cost and mobility (portable devices), but when there are extensive lesions, ultrasonography is less accurate in defining exactly which segments are involved. Additionally, ultrasonography is operator-dependent, which often results in poorly detailed reports and highly generic descriptions of the lesions.

Given the limitations of ultrasonography, abdominal $\mathrm{CT}$ scan has assumed importance as a method for diagnosis and prognosis and also for indicating the best treatment that should be used. The follow-up of disease progression should also use this form of diagnosis (Meneghelli et al. 1986).

E. vogeli cystic lesions are typically round, multiple and hypodense, with a size of approximately $3 \mathrm{~cm}$ (but potentially over $10 \mathrm{~cm}$ ) and a homogeneous content without contrast; peripheral calcification is their main characteristic. These calcifications do not always involve the entire cyst and are irregular and coarse (D'Alessandro \& Raush 2008).

In the cases with hepatic presentation described in this report, the involvement was bilateral in $29.5 \%$ of cases and only $10 \%$ had mesenteric or retroperitoneal involvement only ( 1 case). For patients in whom the involvement was only extrahepatic, it is important to consider whether there is more than one strain of $E$. vogeli, similar to E. granulosus, that may explain the difference in presentation, or whether this finding is the result of a host immune response or simply the result of chance.

Echinococcosis is difficult to treat because of the lack of substantial data in the literature defining the best treatment (Siqueira 2002, D’Alessandro \& Raush 2008). No double-blind and controlled studies are available to guide the best practice. The published cases number less than 200 and most of the publications are case reports; therefore, there is no way to define protocols.

Therefore, treatment is based on the therapy used

\section{TABLE IV}

Duration of follow-up for patients with polycystic echinococcosis included in the study, Rio Branco, state of Acre, 1999-2009

\begin{tabular}{lcccc}
\hline Follow-up (days) & $\begin{array}{c}\text { Albendazole } \\
(\mathrm{n}=29)\end{array}$ & $\begin{array}{c}\text { Surgery } \\
(\mathrm{n}=26)\end{array}$ & $\begin{array}{c}\text { PAIR } \\
(\mathrm{n}=5)\end{array}$ & $\begin{array}{c}\text { Total } \\
(\mathrm{n}=60)\end{array}$ \\
\hline Mean & 771.07 & 761.85 & 638.40 & 884.25 \\
Median & 431.00 & 267.50 & 163.00 & 431.00 \\
Standard deviation & 876.13 & 1.121 .96 & 1.144 .56 & 1.001 .81 \\
Minimum & $0^{a}$ & $0^{a}$ & 39 & $0^{a}$ \\
Maximum & 3.065 & 3.755 & 2.683 & 3.755 \\
\hline
\end{tabular}

$a$ : patients who did not return to the outpatient clinic for reevaluations or who died during hospitalisation. 
for E. granulosus, but because patients are often found in advanced stages of the disease that preclude surgical treatment, which is the gold standard of treatment for cystic echinococcosis, the treatment is based on chemotherapy with benzimidazoles, of which albendazole is the most widely used, at a dose of $10-12 \mathrm{mg} / \mathrm{kg} /$ day (D'Alessandro \& Raush 2008) and on treatment by PAIR (Brunetti et al. 2001). It is therefore to define which patients should receive chemotherapy, puncture therapy or surgical treatment for polycystic echinococcosis.

The PNM classification system (Kern et al. 2006) was proposed for lesions caused by E. multilocularis in alveolar echinococcosis, which is characterised by the replacement of healthy liver tissue by small vesicles that behave similarly to malignant liver tumours, even undergoing metastatic dissemination to distant organs. Polycystic hydatid disease has similar characteristics with respect to hepatic involvement and the possibility of dissemination to other organs. Reports of pulmonary lesions (Ming-Bai et al. 2005) were also included in this study cohort. The applicability of the PNM classification system (Kern et al. 2006) in cases of polycystic echinococcosis was evaluated in this cohort and the PNM system was found to be useful in selecting patients for clinical or surgical treatment. All of the patients with hepatic involvement were classified and ranked into four possible stages, except for patients with exclusively mesenteric involvement. In the these patients, the PNM classification system (Kern et al. 2006) is not applicable because the patients would be classified as stage IIIb or $\mathrm{IV}$, which does not correspond to their actual degree of disease severity. All of these patients underwent surgical treatment with complete excision of their lesions and achieved a cure rate of $100 \%$. Thus, a modification of the PNM classification system (Kern et al. 2006) is proposed in this study, as noted in the Results section, to include the stages "mesenteric" and/or "retroperitoneal".

Many (41\%) patients were at stage IIIb and $52 \%$ of the patients were in the fourth or fifth decade of life, which supports the hypothesis of a long and gradual disease progression.

The patients initially underwent one of two possible forms of therapy: clinical treatment with albendazole or surgical treatment when complete resection of the cysts was possible. Application of the PAIR protocol was supported as an option for unresectable cases, but only for large cysts with a diameter greater than $5 \mathrm{~cm}$.

Treatment by puncture is indicated in cases that present with infected cysts and abscess formation as well as in cases in which surgery is contraindicated because the lesions are not resectable. In all of these cases, the cysts grow to large volumes. These patients benefit the most from percutaneous puncture because efficient drainage of the cyst contents is achieved. In contrast, small lesions with communication between the cysts appear to be a minor hindrance or prevent the complete emptying of lesions. Although incomplete emptying was only observed in a limited number of patients in our study, similar results for PAIR therapy were found for polycystic echinococcosis. A simple fine-needle puncture (standard for E. granulosus cases) did not empty all of the cysts and there was no guarantee that the injected scolicidal agent would reach all of the lesions. Occasionally, the discharge can be thick and contain membrane remnants. Thus, larger-gauge needles (up to $12 \mathrm{~F}$, for example) must be used and these needles cannot be removed immediately after the procedure because of the risk of leakage of the cyst contents into the peritoneal cavity. After aspiration of all cyst contents and injection with the same volume of $10 \%$ sodium chloride solution, the drain is closed for $10 \mathrm{~min}$ and then opened again. It is safer to introduce a shunt of compatible calibre to prevent leakage. In all cases of puncture in our study, there was no uniformity with regard to the duration of time that the shunt should be maintained. This question remains unanswered but is highly relevant, given the possibility for the residual cystic cavities (in cases without prior infection) to show secondary infection and progress to septicaemia. Similarly, the efficacy of antibiotic combinations administered while using the shunt is also unclear for cases without associated bacterial infections.

Video laparoscopy may be useful in assessing the resectability of lesions and in cases of isolated mesenteric or hepatic cysts. This method was used therapeutically by Cantele et al. (2006). Regarding the use of video laparoscopy in cases of polycystic hydatid disease, we observed communication between cysts by injecting iodinated contrast after puncture, but these communications may not be wide enough to empty all of the cysts or to allow scolicidal substances to be injected into all of the cysts. Thus, laparoscopic puncture and irrigation of only one of the cysts, which is then left open to the peritoneum, will inevitably lead to dissemination of the contents of residual cysts into the peritoneal cavity. Video laparoscopy was used in three patients in this cohort for stage classification purposes only. In one patient, video laparoscopy was essential for surgical indication because the resectability of the lesions could not be determined based on the tomographic images.

The hypothesis that benzimidazoles are only parasitostatic (Hemphill et al. 2007) is reinforced by the low rate of "cure" outcomes in the clinical treatment group (4.3\%; $1 / 28)$. The most frequent result obtained in this group was the "clinical improvement" outcome $(60.71 \% ; 17 / 28)$.

The overall patient mortality in this cohort was $15.5 \%(9 / 58)$. Death resulted from portal hypertension complications secondary to the presence of cysts that do not directly invade portal structures in most cases, but still cause hypertension through extrinsic compression. Additionally, biliary fistulas may occur.

Nine of the patients who died were in stage IIIb $(12 \%$; $7 / 58)$, one patient was in stage IV $(1.7 \% ; 1 / 58)$ and one patient was in stage I. In particular, this last patient (stage I) had been treated at the Clinical Hospital of Ribeirão Preto, state of São Paulo for more than 20 years and had undergone five surgical interventions. The patient showed residual lesions (or possible relapse) that were categorised at the time as stage I, but the patient showed clinical signs of portal hypertension. Death occurred at the patient's residence because of massive gastrointestinal bleeding, according to information from the patient's family. The staging of the patient's disease should have been further advanced, but the patient had no access to imaging tests at the time of diagnosis. 
One option for patients in advanced stages may be liver transplantation, particularly for patients whose disease is in stages IIIa, IIIb or IV, involving the hepatic pedicle and preventing any type of liver resection. An en bloc resection is feasible, despite the possible technical difficulties that result from the aggressiveness with which these injuries involve portal structures (Haider et al. 2008).

The five-year survival rate for liver transplants in cases of alveolar echinococcosis is higher than $70 \%$ (Koch et al. 2003). The risk of relapse of alveolar echinococcosis is of concern after transplantation because of immunosuppression (Bresson-Hadni et al. 1991), but in this cohort, there was no case of relapse after surgery with complete resection, which suggests that relapse after immunosuppression is unlikely to occur in cases of E. vogeli echinococcosis without residual lesions.

Notwithstanding these findings, in this study, there was a significant loss of patients to follow-up. Many patients had difficulty returning for medical appointments, despite the guarantee of outpatient care. There were multiple causes: difficulties for patients from the interior of the state in obtaining tickets through the $\mathrm{Mu}-$ nicipal Secretaries of Health, particularly in obtaining airfare during the winter period; financial difficulties related to stays in the capital (which sometimes meant long periods of hospitalisation) and even the perception that their problems had most likely been solved because clinical symptoms regressed quickly after the initiation of clinical treatment.

The regional characteristics of the patients prevent or hinder the bridging of gaps in knowledge of E. vogeli echinococcosis, especially for known indicators and zoonotic cycle elements. In addition, there is a lack of seroepidemiological studies in individuals from Amazonian states and in canine populations from the cities with the greatest number of patients. For E. vogeli echinococcosis, the best indicators of the asymptomatic clinical form must be identified to facilitate early diagnosis. In diagnosed cases, it seems necessary to designate alternative therapies and even plan for, with the consent of the Ethical Committee on Research, the introduction of new chemotherapy treatments to the cyst wall at higher concentrations. Thus, research groups studying tropical diseases, which affect patients in $45 \%$ of Brazilian territory, should work to bridge these and other gaps.

The PNM classification system was useful in identifying cases requiring surgical treatment as well as patients who should remain under clinical treatment. This system may also be used to determine cases that will require liver transplantation.

Surgical treatment produced better results than clinical treatment with respect to "cure" and "clinical improvement" outcomes. Cases with exclusively mesenteric location and those classified as stages I and II had better prognoses and the possibility of complete resection of the lesions.

Patients in stages IIIa, IIIb and IV should be treated with benzimidazoles and re-evaluated for the possibility of surgical resection. If these patients show large cysts (larger than $5 \mathrm{~cm}$ ), they can be treated with PAIR and subsequently with resection if a complete cure of their lesions is not obtained. PAIR treatment, despite being associated with high mortality rates, requires further study because the sample size was small in this study. Patients in stages IIIa, IIIb and IV without surgical indication for cyst resection may require liver transplantation.

\section{REFERENCES}

Acha PN, Szyfres B 1989. Zoonosis y enfermedades transmisibles comunes al hombre y a los animales: hidatidosis, 2nd ed., Organizacion Panamericana de Salud, Washington, p. 734-754.

AJCC - American Joint Committee on Cancer 2008. Cancer staging manual. Available from: cancerstaging.org.

Bresson-Hadni S, Franza A, Miguet JP, Vuitton DA, Lenys D, Monnet E, Landecy G, Paintaud G, Rohmer P, Becker MC, Christophe JL, Mantion G, Gillet M 1991. Orthotopic liver transplantation for incurable alveolar echinococcosis of the liver: report of 17 cases. Hepatology 13: 1061-1070.

Brunetti E, Filice C, Macpherson C, Meslin FX, Vuitton D 2001. PAIR: puncture, aspiration, injection, re-aspiration: an option for the treatment of cystic echinococcosis. Available from: whqlibdoc.who.int/hq/2001/WHO_CDS_CSR_APH_2001.6.pdf.

Cantele HE, Gutiérrez JE, Safar JA, Falco A, Egui MA, Díaz MC, Abdul-Hadi S, Chacón NJ, Bruces AC, Díaz MV, Urdaneta C, Ruíz ME 2006. Tratamiento laparoscópico de un quiste hidatídico hepático poliquístico por Echinococcus vogeli. Reporte de un caso en Venezuela. RFM 29. Available from: scielo.org.ve/scielo. php?pid=S0798-04692006000100010\&script $=$ sci_arttext.

D’Alessandro A 1997. Polycystic echinococcosis in tropical America: Echinococcus vogeli and E. oligarthrus. Acta Trop 67: 43-65.

D’Alessandro A, Raush RL 2008. New aspects of Neotropical polycystic (Echinococcus vogeli) and unicystic (Echinococcus oligarthrus) echinococcosis. Clin Microbiol Rev 21: 380-401.

D’Alessandro A, Raush RL, Cuello C, Aristazabal N 1979. Echinococcus vogeli in man, with a review of polycystic hydatid disease in Colombia and neighboring countries. Am J Trop Med Hyg 28: 303-317.

Guimarães LSC, Chalub SRS, Duarte El, Maia MV, Jaime MV 2005. Cisto hidático - Relato de dois casos no Amazonas. Rev Col Bras Cir 32 (Suppl.): S384.

Haider HH, Nishida S, Selvaggi G, Levi D, Tekin A, Moon JI, Tzakis AG 2008. Alveolar echinococcosis induced liver failure: salvage by liver transplantation in an otherwise uniformly fatal disease. Clin Transplant 22: 664-667.

Hemphill A, Spicher M, Stadelmann B, Muellerj J, Naguleswaran A, Gottstein B, Walker M 2007. Innovative chemotherapeutical treatment options for alveolar and cystic echinococcosis. Parasitology 134: 1657-1670.

Kern P, Wen H, Sato N, Vuitton DA, Gruener B, Shao Y, Delabrousse E, Kratzer W, Bresson-Hadni S 2006. WHO classification of alveolar echinococcosis: principles and application. Parasitol Int 55 (Suppl.): S283-S287.

Koch S, Bresson-Hadni S, Miguet JP, Crumbach JP, Gillet M, Mantion GA, Heyd B, Vuitton DA, Minello A, Kurtz S 2003. Experience of liver transplantation for incurable alveolar echinococcosis: a 45case European collaborative report. Transplantation 75: 856-863.

Meneghelli UG, Barbó MLP, Magro JE, Bellucci AD, Llorach-Velludo MAS 1986. Polycystic hydatid disease (Echinococcus vogeli): clinical and radiological manifestations and treatment whit albendazole of a patient from the Brazilian Amazon Region. Arq Gastroenterol 23: 177-183. 
Meneghelli UG, Martinelli ALC, Velludo MASL 1990. Cistos de Echinococcus vogeli em fígado de paca (Cuniculus paca) originária do estado do Acre, Brasil. Rev Soc Bras Med Trop 23: 153-155.

Ming-Bai W, Li-Wei Z, Hui Z, Zhong-Xi Q 2005. Surgical treatment for thoracic hydatidosis: review of 1,230 cases. Chin Med J 118: $1665-1667$.

Pastore R 2001. Estudo da infecção pelo Echinococcus sp. na área do municipio de Sena Madureira - Acre, MsD Thesis, Universidade Federal de Brasília, Brasília, 161 pp.

Raush RL, Bernstein JJ 1972. Echinococcus vogeli sp. n (Cestoda: Taeniidae) from the bush dog, Speothos venaticus (Lund). $Z$ Prakt Anasth Wiederbeleb Intensivther 23: 25-34.

Raush RL, D'Alessandro A 1999. Histogenesis in the metacestode of Echinococcus vogeli and mechanism of pathogenesis in polycystic hydatid disease. J Parasitol 85: 410-418.

Raush RL, D’Alessandro A, Raush VR 1981. Características de la larva de Echinococcus vogeli Raush y Bersnstein, 1972 en el huésped intermediario natural, la guagua Cuniculus paca L. (Rodentia: Dasyproctidae). Colomb Med 12: 167-175.
Siqueira NG 2002. Doença hidática policística: revisão sistemática da literatura, MsD Thesis, Universidade Federal da Bahia, Salvador, $120 \mathrm{pp}$.

Siqueira NG, Almeida FB, Chalub SRS, Machado-Silva JR, Rodrigues-Silva R 2007. Successful outcome of hepatic polycystic echinococcosis managed with surgery and chemotherapy. Trans R Soc Trop Med Hyg 101: 624-626.

Siqueira NG, Almeida FB, Suzuki YAC, Lima RNA, Machado-Silva JR, Rodrigues-Silva R 2010. Atypical polycystic echinococcosis without liver involvement in Brazilian patients. Trans $R$ Soc Trop Med Hyg 104: 230-233.

Siqueira NG, Santos-Jesus R, Tanajura D, Tavares-Neto J 2003. Doença hidática policística: análise secundária de dados. Rev Col Bras Cir 30: 205-215.

Somocurcio JR, Sánches EL, Náquira C, Schilder J, Rojas F, Chacón P, Yabar A 2004. First report of a human case of polycystics Echinococosis due to Echinococcus vogeli from Neotropical area of Peru, South America. Rev Inst Med Trop Sao Paulo 46: 41-42.

UICC - International Union Against Cancer 2008. TNM classification of malignant tumours. Available from: uicc.org. 
PNM Human Alveolar Echinococcosis Classification System

Parasite liver location $(\mathrm{P})$

PX

P0

P1

$\mathrm{P} 2$

P3

P4

Extrahepatic involvement of neighbouring organs (N) (diaphragm, lung, pleura, pericardium, heart, gastric or duodenal walls, adrenal glands, peritoneum, retroperitoneum, abdominal wall (muscles, skin, bones), pancreas, regional lymph nodes, hepatic ligaments or kidneys)

NX

N0

$\mathrm{N} 1$

\section{Primary lesion cannot be assessed}

Non-detectable liver lesion

Peripheral lesion without vascular and/or biliary involvement

Central lesion with vascular and/or biliary involvement of one lobe ${ }^{a}$
Central lesion with vascular and/or biliary involvement of both lobes or with involvement of two hepatic veins

Any liver lesion with extension along blood vessels ${ }^{b}$ and/or the biliary tree

Absence or presence of distant metastases (M) (lung, distant lymph nodes, spleen, central nervous system, orbit, bones, skin, muscle, distant sites from the peritoneum or retroperitoneum)

$\mathrm{MX}$

M0

M1
Not evaluable

Without metastases ${ }^{c}$

Metastases

$a$ : for classification, the plane projected between the gallbladder bed and inferior vena cava divides the liver into two lobes; $b$ : vessels refer to inferior the vena cava, portal vein and arteries; $c$ : negative lung X-rays and computed tomography of the brain. Source: adapted from Kern et al. (2006).

Staging based on PNM Human Alveolar Echinococcosis Classification System

\begin{tabular}{|c|c|c|c|}
\hline Stage I & $\mathrm{P} 1$ & No & M0 \\
\hline Stage II & $\mathrm{P} 2$ & No & M0 \\
\hline Stage IIIa & $\mathrm{P} 3$ & No & M0 \\
\hline \multirow[t]{2}{*}{ Stage IIIb } & P1-3 & N1 & M0 \\
\hline & P4 & N0 & M0 \\
\hline \multirow[t]{2}{*}{ Stage IV } & $\mathrm{P} 4$ & N1 & M0 \\
\hline & any $\mathrm{P}$ & any $\mathrm{N}$ and/or & M1 \\
\hline
\end{tabular}

source: adapted from Kern et al. (2006). 